\title{
Study of the Transition from Conduction to Injection in an Electrohydrodynamic Flow in Blade-Plane Geometry
}

\author{
Michel Daaboul ${ }^{\mathrm{a}}$, Philippe Traoré ${ }^{\mathrm{b}}$, Pedro Vázquez ${ }^{\mathrm{c}}$, Christophe Louste $^{\mathrm{b}}$ \\ ${ }^{a}$ Mechanical Engineering Department, University of Balamand, Al-Kurah, Lebanon \\ ${ }^{\mathrm{b}}$ EHD Group, Pprime Institute, CNRS, University of Poitiers, ISAE-ENSMA, Poitiers, France \\ ${ }^{c}$ Department of Applied Physics III, University of Seville, Seville, Spain \\ Corresponding author: Michel Daaboul, michel.daaboul@balamand.edu.lb
}

\section{Abstract}

A dielectric fluid can be set into motion with the help of electric forces, mainly Coulomb force. This phenomenon, called electroconvection, can be induced by electrohydrodynamic conduction, injection, and induction. Conduction is based on the dissociation/recombination phenomenon, generates heterocharge layers, and occurs for low electric field values. Injection produces homocharge layers in the electrode vicinity and requires stronger electric fields to be initiated. This study is an experimental observation of the transition from conduction to injection of a dielectric liquid in blade-plane geometry using Particle Image Velocimetry. In addition, the electric current is measured to completely understand the flow behavior.

\section{Keywords}

Conduction; Coulomb Force; Dielectric Liquid; Electrohydrodynamics; Injection; Particle Image Velocimetry.

\section{Introduction}

Electroconvection is the phenomenon of setting a dielectric fluid into motion with the help of electric forces. It is used to produce electrohydrodynamic (EHD) pumps and electroconvective flows as jets, wall jets and impinging jets, etc. Several electric forces can act on a fluid to induce its motion, but Coulomb force is the most important [1]. In order to generate a Coulomb force within a liquid, a volumetric charge density or a space charge must be created. This can be achieved by three main methods: conduction, injection, and induction.

First, in conduction, the electric current is produced by positive and negative ions generated by the dissociation of molecules that come to neutralize the electrodes. Since there is no charge injection at the electrodes, electric charges produced by dissociation are attracted towards the electrode of opposite polarity. They accumulate in the vicinity of that electrode and form what is known as a heterocharge layer [2], since the polarity of this layer is different from that of its adjacent electrode. A force is produced on an electrode and is often countered by a symmetrical 
force of opposite sign on the second electrode. In order to obtain a fluid motion, it is necessary that the produced Coulomb force be asymmetrical on the electrode pair. This is achieved using specific electrode geometries. Conduction, generally, occurs at low values of applied electric field. Many previous studies [2-4] have theoretically, numerically, and experimentally investigated the phenomenon of conduction and its application on EHD pumps. Velocities of the order of $10 \mathrm{~cm} / \mathrm{s}$ were obtained. They also obtained encouraging results for heat transfer and fluid transport applications [6] particularly in insulated applications where other EHD methods are unachievable or undesirable.

The second method is direct injection, which takes place when the electric field reaches a threshold value. It is the process of creating a space charge in the vicinity of the electrodes: electrons are injected into the liquid and bind to neutral molecules to form ions. The accumulation of electric charges results in the appearance of a non-neutral layer composed of ions having the same polarity of the electrode. This is referred to as homocharge layer. Electric charges are then repelled by the electrode. They are set into motion and, due to the fluid viscosity, drive the surrounding liquid. The fact that a dielectric liquid can be moved by ion injection has been known for over a century [7]. Several prototypes of pumps using the injection method (also called ion-drag pumps) were specially designed for cooling applications. The charge injection pressure generation was studied theoretically and experimentally in gases and insulating liquids [8-10]. The injection phenomenon was also studied in EHD pumps and heat transfer enhancement devices [11-12]. Velocities of the order of $1 \mathrm{~m} / \mathrm{s}$ were obtained. The method has also been successfully tested on various dielectric liquids (mainly hydrocarbons). The results obtained depend on the liquid properties including viscosity and electric conductivity [13]. The injection phenomenon has been studied more than the other two methods.

In the induction phenomenon, the space charge is created by a gradient or discontinuity of the electric conductivity. In the presence of an electric field, a volumetric charge density appears in the conductivity gradient zone. This density induced charge is attracted or repelled, thus causing a motion of the liquid. Theoretical and experimental studies were carried out on EHD induction [14-15]. Experimental works have confirmed that induction can also be obtained within a liquid by using a temperature gradient to produce a conductivity gradient [16-17]. The induction process for pumping, flow control applications, and heat transfer enhancement was studied [1821]. The behavior of induction in a condensing liquid was also studied by varying the difference of potential and the frequency of the applied electric signal [22]. The use of EHD induction appears particularly promising for heat transfer applications.

Injection is the technique that has been mostly studied. It is also the most effective. Conduction and induction, although less effective, are also promising for various industrial applications. In practice, in isothermal fluids, conduction and injection can coexist when an electric field is applied, but it is generally assumed that one phenomenon dominates the other one. All these phenomena depend on several parameters such as electrodes geometry, working fluid, operating conditions, etc. 
In this paper, the transition from conduction to injection is presented. Particle Image Velocimetry (PIV) measurements are carried out on a dielectric liquid flow in blade-plane geometry. The applied voltage is increased gradually while current measurements and PIV acquisitions take place simultaneously. This geometry is used because it allows obtaining a classical flow which was commonly studied in EHD [23].

\section{Experimental Setup}

\subsection{Apparatus}

A schematic diagram of the experimental apparatus is shown in Figure 1. It consists of two copper plane electrodes (1) and (2). The dimensions of each electrode are $60 \mathrm{~mm} \times 45 \mathrm{~mm} \times 1 \mathrm{~mm}$ and their ends are rounded with a radius of curvature of $0.5 \mathrm{~mm}$. The first electrode (1) is connected to a Spellman SL100 positive DC power supply providing a voltage going from $0 \mathrm{~V}$ up to $+5 \mathrm{kV}$. It is referred to as the blade and is in a horizontal position. The second electrode (2) is grounded and is referred to as the counter-electrode in the following sections of this paper. It is placed vertically in front of the blade with an inter-electrode distance of $1 \mathrm{~cm}$. These two electrodes are placed in an $80 \mathrm{~mm} \times 65 \mathrm{~mm} \times 40 \mathrm{~mm}$ cavity cell (3) made of PMMA. In order to avoid edge effects, the electrode edges are installed in two sidewalls (4) and (5) also made of PMMA. The cell is filled with the dielectric liquid through two holes (6) located on the upper surface of the cell so that air bubbles are evacuated. Two sealing strips (7) are placed in the two sidewalls to avoid leakage.

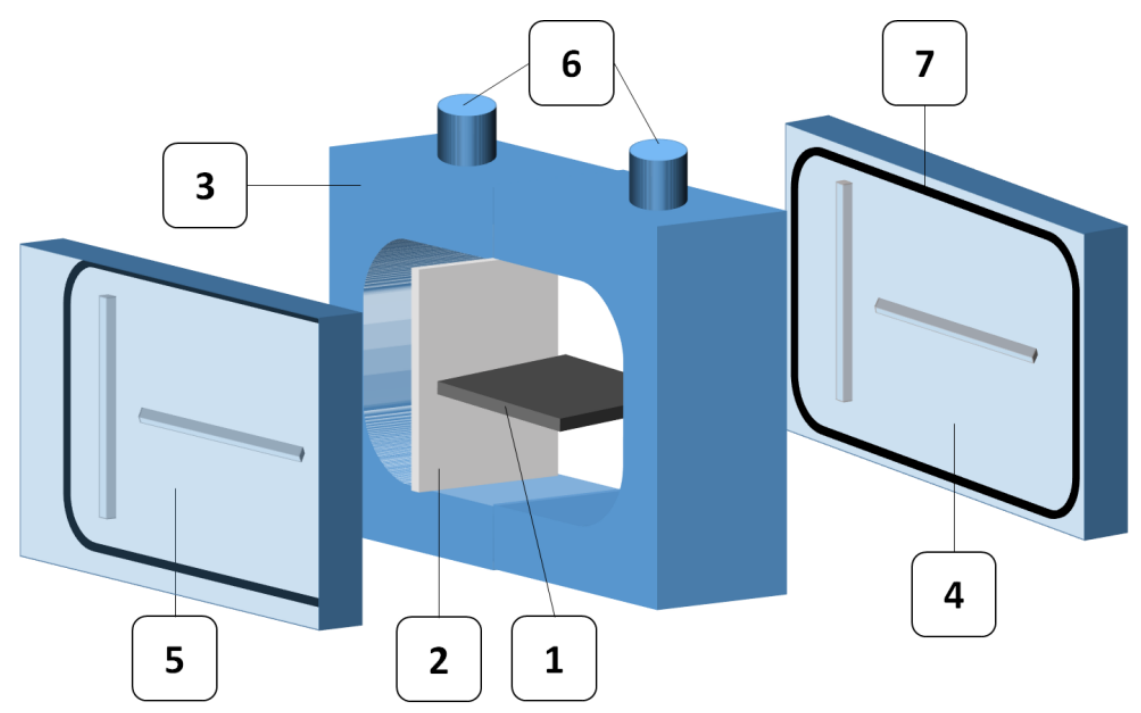

Figure 1. Experimental apparatus. 


\subsection{Dielectric Liquid}

The dielectric liquid used to conduct the experiments of this work is $3 \mathrm{M}^{\mathrm{TM}}$ Novec $^{\mathrm{TM}}$ Engineered Fluid HFE-7100 whose properties at ambient temperature are presented in Table 1.

Table 1. Properties of HFE-7100 at a temperature of $25^{\circ} \mathrm{C}[24]$.

\begin{tabular}{|l|l|l|}
\hline Mass density & $\rho$ & $1520 \mathrm{~kg} / \mathrm{m}^{3}$ \\
\hline Kinematic viscosity & $v$ & $3.9 \times 10^{-1} \mathrm{~m}^{2} / \mathrm{s}$ \\
\hline Dynamic viscosity & $\mu$ & $5.9 \times 10^{-4} \mathrm{~Pa} . \mathrm{s}$ \\
\hline Electric conductivity & $\sigma$ & $10^{-6} \mathrm{~S} / \mathrm{m}$ \\
\hline Dielectric strength & $E_{S}$ & $16 \mathrm{kV} / \mathrm{mm}$ \\
\hline Relative permittivity & $\varepsilon$ & 7.4 \\
\hline
\end{tabular}

\subsection{Particle Image Velocimetry System}

The PIV technique is a measurement method that records complete velocity fields of a desired flow configuration. A schematic is shown in Figure 2. In this work, a LaVision PIV system (LaVision GmbH, Göttingen, Germany) was used. A Laser sheet illuminated the scene, which is seeded by very small tracer particles, and a CCD camera recorded successive images of the flow at a spatial resolution of $1376 \times 1040$ pixels. The software used to analyze PIV images is Davis 8.0. The velocity fields were then calculated by cross-correlation or time series with windows of $32 \times 32$ pixels.

\section{Flow}

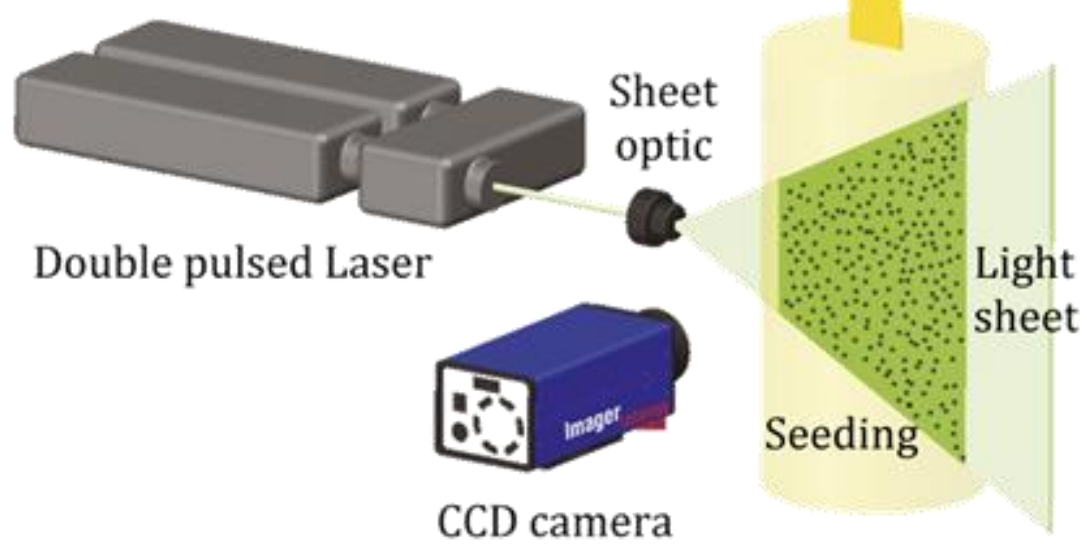

Figure 2. Particle Image Velocimetry system (C LaVision GmbH, Germany, www.lavision.de)

\subsection{Seeding Particles}

In order to obtain relevant PIV images, the fluid must be seeded with very small particles. Nevertheless, their concentration must not exceed a certain limit [25]. The properties of these particles must be compatible with the properties of the dielectric fluid. It must have a close mass density to avoid floatation and sedimentation along with close dielectric properties to limit particle charging. The particles used in this work are made of PTFE and have a diameter of less than $1 \mu \mathrm{m}$. Their properties are shown in Table 2 . 
Table 2. Properties of PTFE at a temperature of $25{ }^{\circ} \mathrm{C}$ [24].

\begin{tabular}{|l|l|l|}
\hline Mass density & $\rho$ & $2200 \mathrm{~kg} / \mathrm{m}^{3}$ \\
\hline Electric conductivity & $\sigma$ & $<10^{-16} \mathrm{~S} / \mathrm{m}$ \\
\hline Relative permittivity & $\varepsilon$ & 2.1 \\
\hline
\end{tabular}

\section{Results}

Before presenting the experimental results, a quick simulation of the applied electric field in the chamber was carried out on Ansoft Maxwell software. In Figure 3, the electric field for a unit difference of potential between the electrodes is shown. It is evident that the highest electric field is obtained on the blade tip. That means the flow will be directed towards the tip in the case of conduction pumping and from the tip towards the counter-electrode in the case of injection pumping.

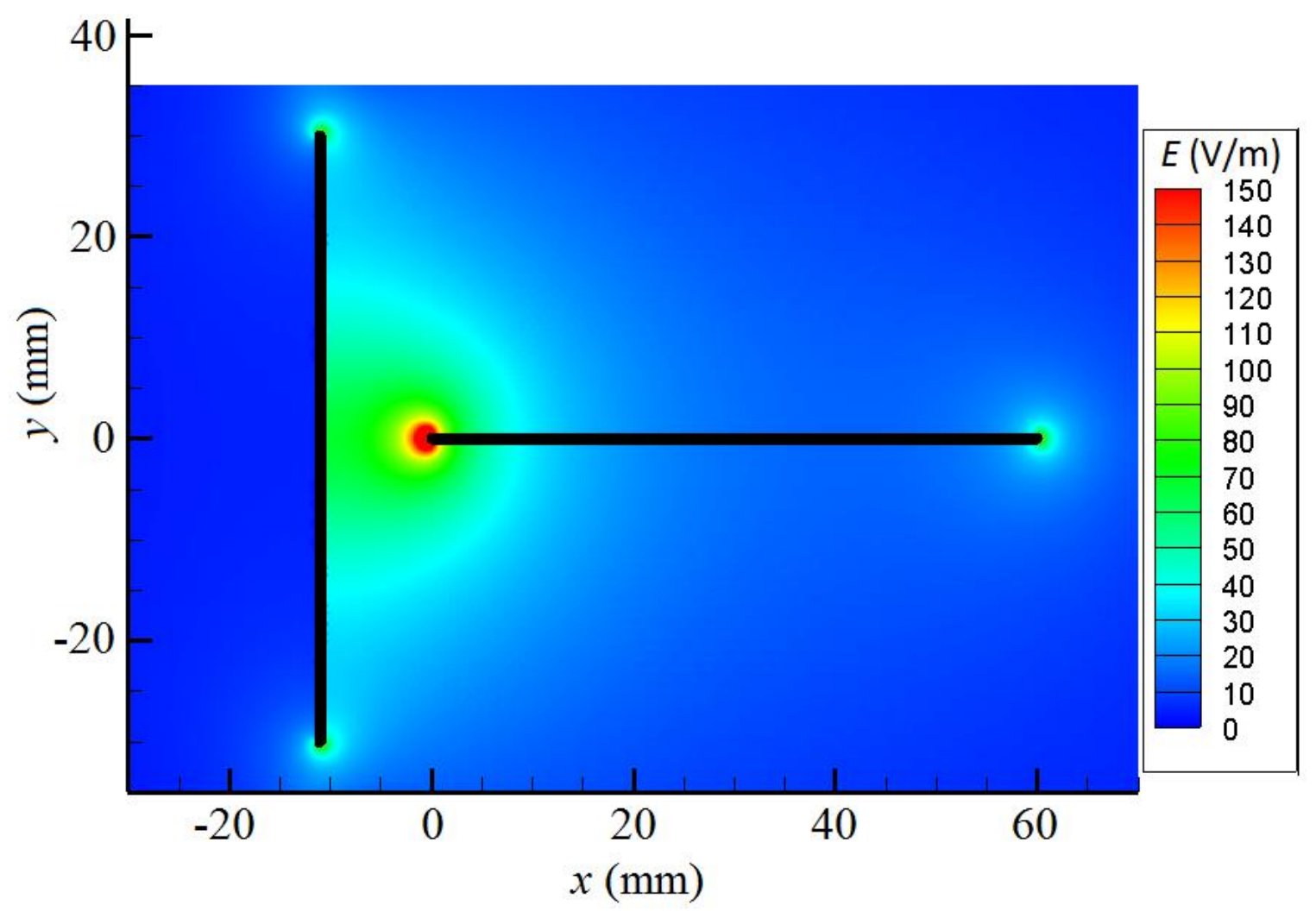

Figure 3. Electric field distribution in the chamber.

The experiments were carried out on several days at a local temperature of around $30{ }^{\circ} \mathrm{C}$. The current/voltage characteristics were measured while the PIV images were taken. A set of 1000 images was recorded for each different value of the applied voltage between the electrodes. Because the flow is mostly unsteady, the average velocity field was then calculated for the 1000 images and the results presented here are based on average values. 


\subsection{Current/Voltage Characteristics}

In order to measure the current/voltage characteristics, the voltage was varied from $0 \mathrm{~V}$ up to $+5 \mathrm{kV}$ on the horizontal electrode while the vertical counter-electrode was always grounded. A shunt resistance was connected in series with the counter-electrode and a LECROY WS424 oscilloscope recorded the corresponding voltage. The current was then calculated and the results are presented in Figure 4.

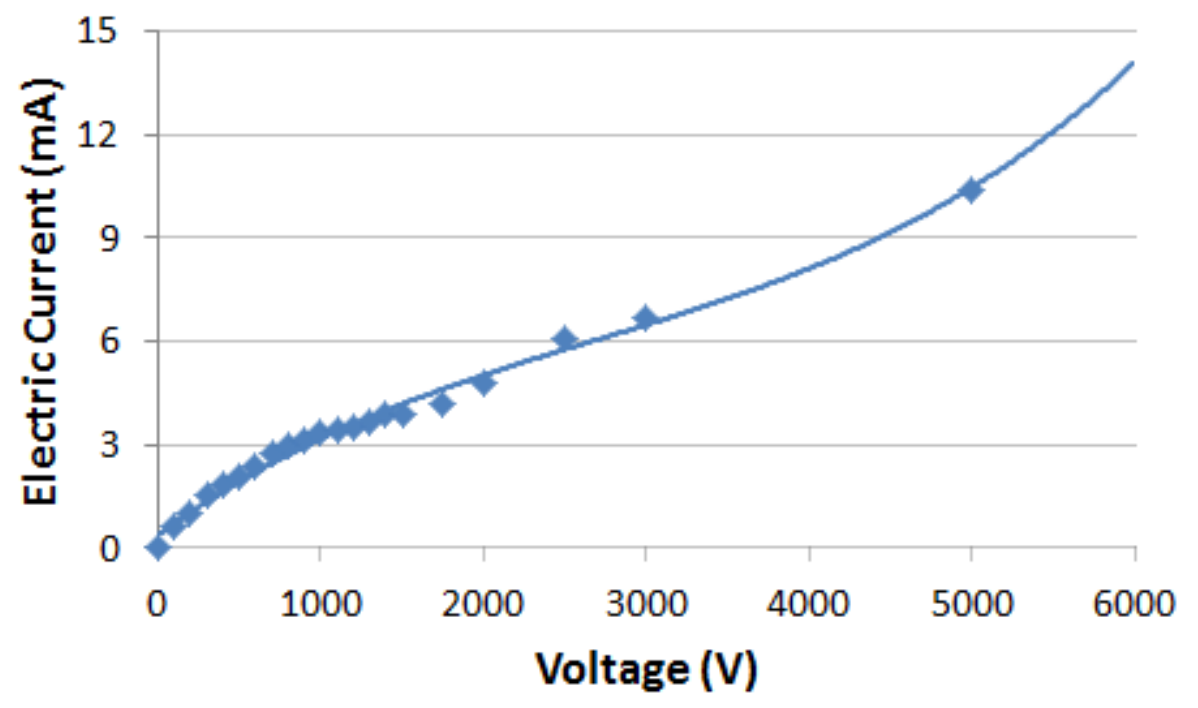

Figure 4. Current/voltage curve.

It can be observed that the current curve starts increasing almost linearly with the applied voltage, for voltages below $1000 \mathrm{~V}$. It is the ohmic region depending on the conductivity of the fluid. This behavior is a characteristic of EHD conduction in such configurations. Between $1000 \mathrm{~V}$ and $2000 \mathrm{~V}$, a transition behavior is seen, remaining linear but with a lower slope. This is the quasi-ohmic region, also known as the saturation region, governed by conduction but affected by the change of the dissociation rate. Beyond $2000 \mathrm{~V}$, an exponential behavior is observed and is characterized by ions injection in the chamber. Note that the electric current was not always steady during measurements, average values were also considered. A PIV study is now required to validate the conclusions made on the electrical behavior.

\subsection{PIV Velocity Fields}

For a better understanding of the behavior of the dielectric liquid when exposed to a high voltage in blade-plane geometry, PIV measurements are necessary. They provide global velocity fields of the study zone and allow investigating the behavior in detail. They also offer the possibility of confirming the deductions made on the electrical behavior. PIV measurements were carried out for voltages going from $0 \mathrm{~V}$ to $5000 \mathrm{~V}$ with an increment of only $100 \mathrm{~V}$ in some ranges. In Figure 5, the velocity vectors of the flow are shown for just four values of the applied voltage. In the background, the velocity contour is seen. These results are the averages of 1000 instantaneous images taken for each case. 


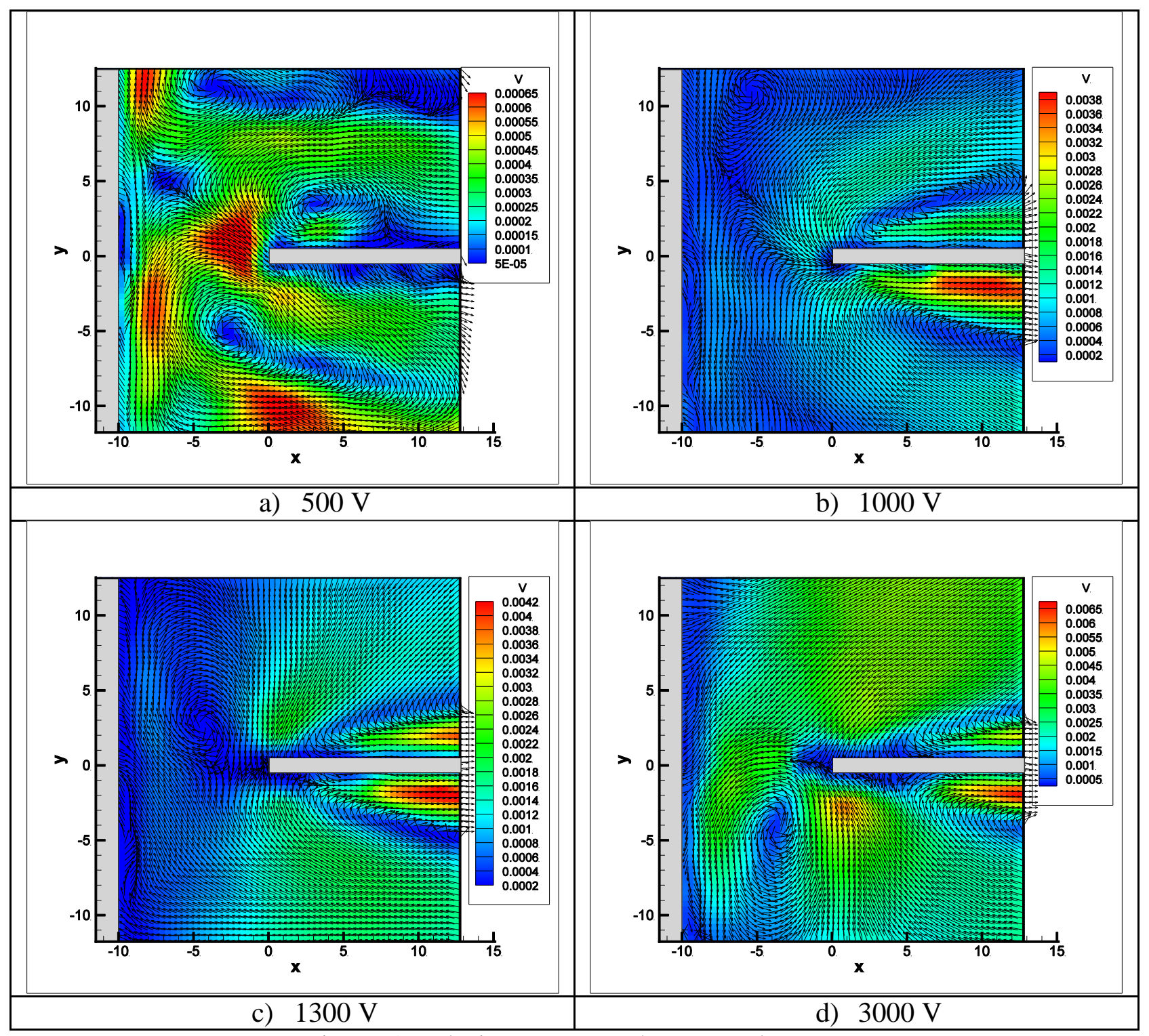

Figure 5. Velocity vectors and contours by PIV.

It can be observed that, for low voltages (Figure 5a), a flow is generated and is directed towards the blade tip. This can be explained by the conduction phenomenon which creates an EHD force towards the electrode with the lowest radius of curvature, which is the blade here. The main flow creates vortices due to mass conservation. Velocities vary from around $1 \mathrm{~mm} / \mathrm{s}$ at $500 \mathrm{~V}$ to $4 \mathrm{~mm} / \mathrm{s}$ at $1000 \mathrm{~V}$. It is also believed that injection occurs simultaneously but is probably negligible compared to conduction. When the applied voltage exceeds $1300 \mathrm{~V}$ (Figure 5c), the direction of the flow is reversed. A jet is generated at the blade tip and flows towards the counter-electrode where it impacts to create two wall jets (up and down). Due to the viscosity of the fluid, two vortices are created. Their direction of rotation is opposite to the direction of previous vortices observed for conduction cases. Similarly, it is thought that conduction phenomenon still exists but is now negligible compared to injection. This can probably explain the three-dimensional behavior of the flow which was clearly seen on instantaneous PIV images. 
Velocities reached values from $4 \mathrm{~mm} / \mathrm{s}$ up to $1 \mathrm{~cm} / \mathrm{s}$ at $5000 \mathrm{~V}$. An important behavior was also observed: 1000 images were not sufficient to obtain a symmetrical flow between the top and the bottom of the chamber. It is clear that a higher number of images is required to obtain smoother average velocity fields, mainly at high voltages where charge injection is more important. This was the case with other fluids previously studied [13],[26].

\section{Discussion}

After carrying out PIV measurements on the EHD flow, both conduction and injection phenomena were seen in the chamber. In this section, a thorough analysis and comparison are presented in terms of the axial velocity. This term is used to define the horizontal velocity on the axis of the blade in the region between the middle point of the counter-electrode and the blade tip. In Figure 6, the behavior of the axial velocity at the symmetry axis of the flow is outlined for various values of the applied voltage.

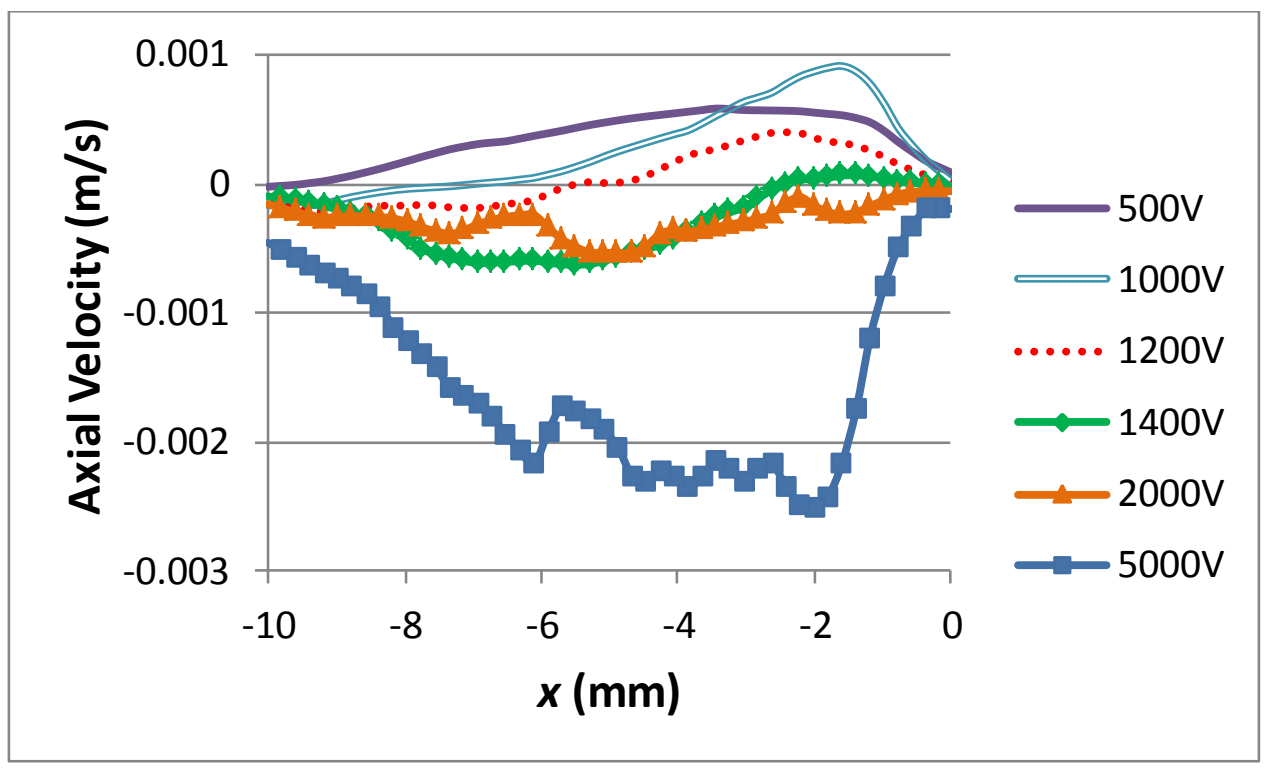

Figure 6. Axial velocity on the symmetry axis of the flow.

One can clearly observe that the axial velocity takes positive and negative values. Positive values are recorded when the main flow is directed towards the blade tip, and therefore shows a dominance of EHD conduction. This is true for voltages below $1200 \mathrm{~V}$. Negative axial velocities exist when the flow is generated at the blade tip and is directed towards the counter-electrode. In this case, injection is dominant and is seen for voltages exceeding $1400 \mathrm{~V}$. The transition from conduction to injection is not quite clear and does not occur at a single threshold value of the applied voltage. On the contrary, there is a transition region where both phenomena exist altogether and no one is able to dominate over the other. This can be explained by velocities of the same order of magnitude for voltages between $1400 \mathrm{~V}$ and $2000 \mathrm{~V}$. Beyond $2000 \mathrm{~V}$, it is clear that EHD injection is the main source of the flow. 


\section{Conclusion}

In this paper, the behavior of a dielectric liquid exposed to an electric voltage in blade-plane geometry was presented. A positive DC voltage up to $5 \mathrm{kV}$ was applied between two perpendicular electrodes. Two main electrohydrodynamic phenomena exist simultaneously: conduction and injection. A different flow was induced in the dielectric liquid for each case.

At low voltages below $1200 \mathrm{~V}$, the heterocharge layer on the electrodes is dominant. Conduction phenomenon occurs in the fluid and leads to the formation of heterocharge layers in the vicinity of the electrodes. A fluid flow is induced within the chamber and is directed towards the blade tip. Due to mass conservation, it creates two contra-rotating vortices which direction proved that injection is neglected.

For voltages beyond $1.4 \mathrm{kV}$, injection is dominant. The homocharge layer becomes important at the blade tip and unipolar injection occurs to induce a flow towards the counter-electrode. Similarly, due to mass conservation, two contra-rotating vortices are created. However, their direction is inverted because of the opposite direction of the main flow which proves that it is the case of ion-drag pumping.

The transition from EHD conduction to injection does not occur suddenly. A transition range is observed between $1200 \mathrm{~V}$ and $2000 \mathrm{~V}$. In this range, both phenomena coexist and the flow is unsteady as clearly shown in the PIV images.

Finally, it is very important to study the behavior of the fluid for negative voltages and carry out a comparison between both signal polarities. Preliminary tests were conducted and different results were observed. This will constitute the subject of a future work.

\section{References}

[1] Hanaoka R., Takata S., Murakumo M., Anzai H., Properties of liquid jet induced by electrohydrodynamic pumping in dielectric liquids, Electrical Engineering in Japan, Vol. 138, Issue 4, pp.1-9, 2002.

[2] Feng Y., Seyed-Yagoobi J., Understanding of electrohydrodynamic conduction pumping phenomenon, Physics of Fluids, Vol. 16, pp.2432-2441, 2004.

[3] Atten P., Seyed-Yagoobi J., Electrohydrodynamically induced dielectric Liquid Flow through Pure Conduction in Point/Plane Geometry, IEEE Transactions on Dielectrics and Electrical Insulation, Vol. 10, pp.27-36, 2003.

[4] Jeong S.I., Seyed-Yagoobi J., Experimental study of electrohydrodynamic pumping through conduction phenomenon, Journal of Electrostatics, Vol. 56, pp.123-133, 2002. 
[5] Jeong S.I., Seyed-Yagoobi J., Atten P., Theoretical/numerical study of electrohydrodynamic pumping through pure conduction phenomenon, IEEE Transactions on Industry Applications, Vol. 39, pp.355-361, 2003.

[6] Siddiqui M.A.W., Seyed-Yagoobi J., Experimental study of pumping of liquid film with electric conduction phenomenon, IEEE Transactions on Industry Applications, Vol. 45, Issue 1, pp.3-9, 2009.

[7] Chattok A.P., Walker W.E., Dixon E.H., Philosophical Magazine, Vol. 1, p.79, 1901.

[8] Stuetzer O.M., Ion-drag pressure generation, Journal of Applied Physics, Vol. 30, pp.984994, 1959.

[9] Pickard W.F., Ion-drag pumping I - theory, Journal of Applied Physics, Vol. 34, pp.246-250, 1963.

[10] Pickard W.F., Ion-drag pumping II - experiments, Journal of Applied Physics, Vol. 34, pp.251-258, 1963.

[11] Sharbaugh A.H., Walker G.W., The design and evaluation of an ion-drag dielectric pump to enhance cooling in a small oil-filled transformer, IEEE Transactions on Industry Applications, Vol. 21, pp.950-955, 1985.

[12] Bryan J.E., Seyed-Yagoobi J., An experimental investigation of ion-drag pump in a vertical and axisymmetric configuration, IEEE Transactions on Industry Applications, Vol. 28, pp.310316, 1992.

[13] Daaboul M., Louste C., Romat H., Transient velocity induced by electric injection in bladeplane geometry, Journal of Electrostatics, Vol. 67, Issues 2-3, pp.359-364, 2009.

[14] Melcher J.R., Traveling wave induced electro-convection, Physics of Fluids, Vol. 9, pp.1548-1555, 1966.

[15] Melcher J.R., Firebaugh M.S., Traveling wave bulk electroconvection induced across a temperature gradient, Physics of Fluids, Vol. 10, pp.1178-1185, 1967.

[16] Wong J., Melcher J.R., Thermally induced electroconvection, Physics of Fluids, Vol. 12, pp.2264-2269, 1969.

[17] Kervin D.J., Crowley J.M., Krein P., Chato J.C., Parametric studies of a large thermal EHD induction pump, Conference Record, IEEE Industry Applications Society Annual Meeting, New York, pp.1015-1020, 1981. 
[18] Seyed-Yagoobi J., Chato J.C., Crowley J.M., Krein P., Induction electrohydrodynamic pump in a vertical configuration, part 1 - theory, ASME Journal of Heat Transfer, Vol. 111, pp.664-669, 1989.

[19] Seyed-Yagoobi J., Chato J.C., Crowley J.M., Krein P., Induction electrohydrodynamic pump in a vertical configuration, part 2 - experimental study, ASME Journal of Heat Transfer, Vol. 111, pp.670-674, 1989.

[20] Wawzyniak M., Seyed-Yagoobi J., Morrison G.L., An experimental study of electrohydrodynamic induction pumping of a stratified liquid/vapor medium, ASME Journal of Heat Transfer, Vol. 122, pp.200-203, 2000.

[21] Wawzyniak M., Seyed-Yagoobi J., Electrohydrodynamic induction pumping of a stratified liquid/vapor medium in the presence of volumetric and interface electric charges, IEEE Transactions on Industry Applications, Vol. 37, pp.950-958, 2001.

[22] Brand K., Seyed-Yagoobi J., Experimental study of electrohydrodynamic induction pumping of a dielectric micro liquid film in external horizontal condensation process, ASME Journal of Heat Transfer, Vol. 125, pp.1096-1105, 2003.

[23] McCluskey F.M.J., Perez A.T., The electrohydrodynamic plume between a line source of ions a flat plate, IEEE Transactions on Electrical Insulation, Vol. 27, No. 2, pp.334-341, 1992.

[24] 3M ${ }^{\mathrm{TM}}$ Specialty Materials, www.3m.com, retrieved on October 16, 2016.

[25] Daaboul M., Louste C., Romat H., PIV measurements on charged plumes - Influence of $\mathrm{SiO}_{2}$ seeding particles on the electrical behavior, IEEE Transactions on Dielectrics and Electrical Insulation, Vol. 16, No. 2, pp.335-342, 2009.

[26] Daaboul M., Louste C., Romat H., PIV Measurements of the Influence of Seeding Particles Concentration on the Velocity of an EHD Flow, 2009 Electrostatics Joint Conference, Boston USA, June 16-18, 2009. 